\title{
Dual innervation of white adipose tissue: some evidence for parasympathetic nervous system involvement
}

\author{
Timothy J. Bartness \\ Neurobiology and Behavior Program, Department of Biology and Center for Behavioral Neuroscience, Georgia State University, \\ Atlanta, Georgia, USA \\ J. Clin. Invest. 110:1235-1237 (2002). doi:10.1172/JCI200217047.
}

The regulation of total body fat was first formally suggested in the lipostatic theory of Kennedy (1), and since that time, the mechanisms underlying the control of lipid energy stores have been sought. The waxing and waning of circulating factors that promote lipid storage or mobilization (e.g., insulin and adrenal medullary catecholamines, respectively) may be the method by which the brain adjusts metabolism as part of a feedback system regulating body fat levels. In other systems, fine and rapid control of biological responses requires direct neural involvement. For example, cardiac function is regulated in this manner through the opposing effects of its innervation by the two arms of the autonomic nervous system: the sympathetic (SNS) and parasympathetic (PSNS) nervous systems. Dual autonomic nervous system innervation of peripheral tissues is the rule, except for sweat glands, which are thought only to have SNS innervation, but until now, such dual innervation has not been shown for either brown adipose tissue or white adipose tissue (WAT). In this issue of the JCI, Kreier et al. (2) present neuroanatomical and functional evidence for the PSNS innervation of WAT, which complements the

\footnotetext{
Address correspondence to: Timothy J. Bartness, Department of Biology, MSC 8L0389, Georgia State University, 33 Gilmer Street SE, Unit 8, Atlanta, Georgia 30303-3088, USA.

Phone (404) 651-2766; Fax: (404) 651-2509;

E-mail: bartness@gsu.edu.

Conflict of interest: No conflict of interest has been declared.

Nonstandard abbreviations used:

sympathetic nervous system (SNS);

parasympathetic nervous system (PSNS);

white adipose tissue (WAT);

pseudorabies virus (PRV).
}

recent substantiation of the SNS innervation of this tissue $(3,4)$. Given the general lack of appreciation of the innervation of WAT until recently, despite many years of supportive functional and neuroanatomical evidence (for review see ref. 5), some discussion of WAT innervation seems warranted.

\section{Sympathetic innervation of WAT}

The development of the Falck-Hillarp histofluorescence technique in the early 1960s permitted clear classification of nerves innervating WAT as catecholaminergic (6). Some controversy initially existed, however, as to whether these nerves supplied WAT blood vessels, parenchyma, or both. Soon thereafter both vascular and parenchymal innervation were identified (7). The advent of modern tract tracing methodologies has provided further, unequivocal identification of this WAT innervation as sympathetic. For example, we demonstrated the sympathetic postganglionic neuronal innervation of WAT in Siberian hamsters and in laboratory rats using fluorescent tract tracers from both anterograde and retrograde directions (4).

One key role of the SNS innervation of WAT is to trigger lipolysis, a catabolic function. Kreier et al. (2) suggest that the role of the PSNS innervation of WAT is to promote lipid accumulation - an anabolic function. The demonstration that adrenal demedullation does not block fat mobilization (8) and that the sympathetic drive to WAT (norepinephrine turnover) is increased by conditions that stimulate lipolysis [e.g., fasting (ref. 9) and cold exposure (ref. 10)] has provided functional evidence that lipolysis by white adipocytes is triggered primarily by norepinephrine released from sympathetic nerve terminals. The neuroanatomical evidence discussed above also reinforces this functional evidence of SNS innervation of WAT.

\section{Using viruses to define neuronal pathways}

The development of viral tract tracers, such as the Bartha $K$ strain of the pseudorabies virus (PRV), in order to define entire neural circuits within the same animal made possible the identification of the CNS circuitry from the brain to peripheral tissues (11). This methodology was adopted in our laboratory (3) and in the present study by Kreier et al. (2) for use in determining the CNS origins of the autonomic outflow to WAT (Figure 1). Briefly, PRV is injected into the tissue of interest (WAT in this case), and the virions are endocytosed into neurons and transported retrogradely to the soma, where viral replication occurs. Virions leave the neurons through the dendrites and infect all neurons with which they make synaptic contact. This process continues, causing an infection along a hierarchical chain of functionally connected neurons, thereby defining neural circuits that project to the injection site from the brain (11). With the availability of genetic mutants of the virus, multiple target sites can be injected to test for convergence and divergence of neural circuits. For example, in the present study Kreier et al. (2) tested for convergence of the SNS innervation of WAT using two white fat depots (a subcutaneous and an intraperitoneal WAT pad). Infected neurons are visualized using standard immunocytochemical techniques or by using PRV mutants with reporters for markers. Because transfer of the 


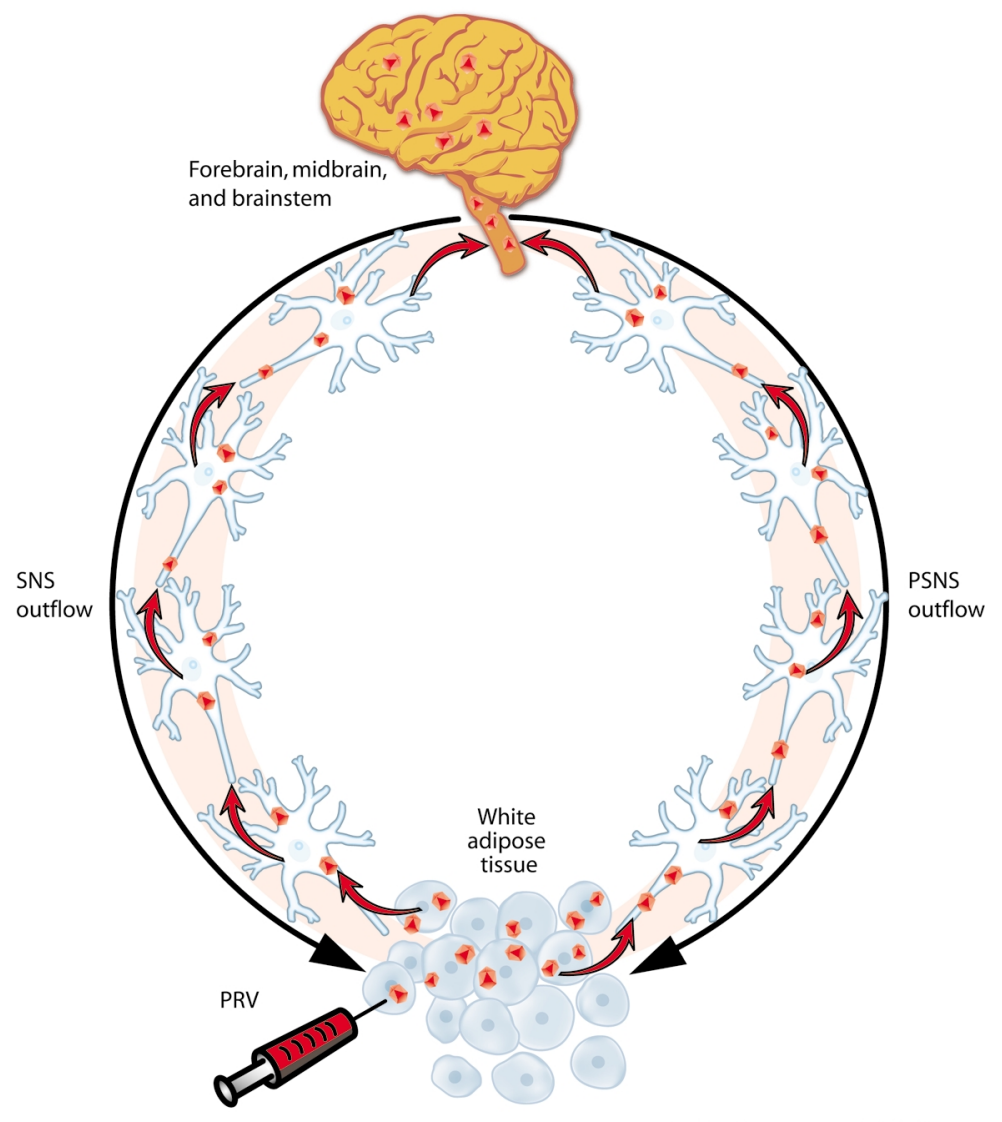

by Ken Beauchamp J. Clin. Invest.

Figure 1

Schematic representation of how the pseudorabies virus (PRV; red dots) retrogradely labels (red arrows) the sympathetic nervous system (SNS; black arrow, left side) and the parasympathetic nervous system (PSNS; black arrow, right side) outflow from the brain to the white adipose tissue.

virus is only via synaptic contact and not by lateral spread to adjacent, but unrelated, neurons, PRV tract tracing provides the high degree of specificity needed to define complete neural circuits $(11,12)$.

\section{Parasympathetic innervation of WAT}

Before this work described by Kreier et al. (2), there was little or no evidence for the PSNS innervation of WAT. For example, the activity of acetylcholinesterase, a necessary enzyme for catabolism of the principal postganglionic neurotransmitter of the PSNS - acetylcholine - is undetectable in $\operatorname{WAT}(13,14)$. In our study of the SNS inputs to WAT using the PRV (3), we noticed that some infected neurons were scattered among the cells of the dorsal motor complex of the brainstem, but were unsure as to whether they were sympathetic neurons found in a brain region traditionally considered as parasympathetic or were part of a parasympathetic outflow to WAT - we chose the former. In contrast, Kreier et al. (2) found substantial infections of neurons in the dorsal vagal complex. This was most likely due to the local surgically induced sympathectomy of WAT before PRV injection, which may have permitted the PSNS drive on WAT to go unchecked, thereby facilitating virus uptake and/or transport and thus highlighting the PSNS innervation. Indisputable confirmation of the parasympathetic nature of this innervation at the level of the postganglionic neurons needs to be provided by colocalization of PRV with a PSNS marker or functionally via electrical stimulation studies where lipolysis would not be stimulated, but the uptake of glucose and/or free fatty acids would be stimulated.

Functionally, Kreier et al. (2) demonstrated that the local PSNS denervation impressively reduced insulindependent uptake of circulating glucose and free fatty acids, and increased the activity of hormone-sensitive lipase - the enzyme responsible for triggering intracellular triglyceride breakdown. Because paired WAT pads are each innervated independently, demonstration of local PSNS denervation was achieved by surgically removing the PSNS innervation of WAT on one side of the animal (verified by viral injection of the denervated tissue yielding no observation of infected neurons in the dorsal vagal complex), with the contralateral side serving as a within-animal control Therefore, by inference, these results suggest that the PSNS innervation of WAT has an anabolic function - the promotion of lipid accumulation.

Finally, Kreier et al. (2) suggested that the SNS innervation of WAT is organized in a viscerotopic manner. We previously reported that the postganglionic sympathetic neurons projecting to the inguinal and epididymal WAT pads were largely located at different levels of the sympathetic chain (4). Here, Kreier et al. (2) extended this finding by injecting two strains of the PRV, one each into mesenteric and inguinal WAT. This resulted in viral labeling from both injection sites in the intermediolateral horn of the spinal cord, but not in the same preganglionic sympathetic neurons, thereby providing anatomical support for depotspecific differences in the lipolytic rate among WAT pads $(15,16)$.

Collectively, the results of the present study by Kreier et al. (2) suggest the PSNS innervation of WAT, and the authors indicate that this innervation may play a significant role in the regional specificity of fat pad metabolism, especially lipid accumulation. Although this is purely speculative, it may be that visceral fat is innervated to a greater degree, or has greater PSNS drive, than non-visceral fat. This could result in enhanced regional accumulation of lipid with certain pathologies, such as Cushing syndrome. This expansion of the adipose organ in these areas could lead to several well-known adverse health consequences of enhanced visceral/ abdominal adiposity such as type II diabetes, certain cancers, and high blood pressure. Alternatively, the decreases in SNS drive seen with many obesities could aid in lipid accumulation by diminishing basal 
and stimulated lipolysis. Moreover, this enhanced lipid accrection could, if Kreier et al. (2) are correct, be further augmented by the anabolic function of the PSNS innervation of WAT. Further neuroanatomical and functional studies are required, however, before we can state with certainty that the autonomic phenotype and function of the nerves described by Kreier et al. (2) are parasympathetic and participate in nonpathological, naturally occurring lipid accretion or, alternatively, are associated with pathological obesity states.

1. Kennedy, G.C. 1953. The role of depot fat in the hypothalamic control of food intake in the rat. Proceedings of the Royal Society of London. B. 140:578-592.

2. Kreier, F, et al. 2002. Selective parasympathetic innervation of subcutaneous and intra-abdominal fat - functional implications. J. Clin. Invest.
110:1243-1250. doi:10.1172/JCI200215736.

3. Bamshad, M., et al. 1998. Central nervous system origins of the sympathetic nervous system outflow to white adipose tissue. Am. J. Physiol. 275:R291-R299.

4. Youngstrom, T.G., and Bartness, T.J. 1995. Catecholaminergic innervation of white adipose tissue in the Siberian hamster. Am. J. Physiol. 268:R744-R751.

5. Bartness, T.J., and Bamshad, M. 1998. Innervation of mammalian white adipose tissue: Implications for the regulation of total body fat. Am. J. Physiol. 275:R1399-R1411.

6. Daniel, H., and Derry, D.M. 1969. Criteria for differentiation of brown and white fat in the rat. Can J. Physiol. Pharmacol. 47:941-945.

7. Slavin, B.G., and Ballard, K.W. 1978. Morphological studies on the adrenergic innervation of white adipose tissue. Anat. Rec. 191:377-389.

8. Kumon, A., Takahashi, A., Hara, T., and Shimazu, T. 1976. Mechanism of lipolysis induced by elec trical stimulation of the hypothalamus in the rabbit. J. Lipid Res. 17:551-558.

9. Migliorini, R.H., Garofalo, M.A.R., and Kettelhut, I.C. 1997. Increased sympathetic activity in rat white adipose tissue during prolonged fasting. Am. J. Physiol. 272:R656-R661.
10. Garofalo, M.A.R., Kettelhut, I.C., Roselino, J.E.S. and Migliorini, R.H. 1996. Effect of acute cold exposure on norepinephrine turnover rates in rat white adipose tissue. J. Auton. Nerv. Syst. 60:206-208.

11. Card, J.P., et al. 1990. Neurotropic properties of pseudorabies virus: uptake and transneuronal passage in the rat central nervous system. J. Neurosci. 10:1974-1994.

12. Strack, A.M., and Loewy, A.D. 1990. Pseudorabies virus: a highly specific transneuronal cell body marker in the sympathetic nervous system. J. Neu rosci. 10:2139-2147.

13. Ballantyne, B. 1968. Histochemical and biochemical aspects of cholinesterase activity of adipose tissue. Arch. Int. Pharmacodyn. Ther. 173:343-350.

14. Ballantyne, B., and Raftery, A.T. 1974. The intrinsic autonomic innervation of white adipose tissue. Cytobios. 10:187-197.

15. Bartness, T.J., Hamilton, J.M., Wade, G.N., and Goldman, B.D. 1989. Regional differences in fat pad responses to short days in Siberian hamsters. Am. J. Physiol. 257:R1533-R1540.

16. Sztalryd, C., and Kraemer, F.B. 1994. Differences in hormone-sensitive lipase expression in white adipose tissue from various anatomic locations of the rat. Metabolism. 43:241-247. 